\title{
TOMOON: A Novel Approach for Topology-Aware Overlay Multicasting
}

\author{
Xiao Chen, Huagang Shao, and Weinong Wang \\ Regional Network Center of East China, \\ Department of Computer Science and Technology, \\ Shanghai Jiao Tong University, China \\ \{shawn, hgshao, wnwang\}@sjtu.edu.cn
}

\begin{abstract}
Most existing overlay multicast approaches avoid considering any network layer support no matter whether it is available or not. This design principle greatly increases the complexity of the routing algorithms and makes the overlay topologies incompatible with the underlying network. To address these issues, we propose TOMOON as a novel overlay multicast approach, which exploits the cooperation between endhosts and IP multicast routers to construct a topology-aware overlay tree. Through a little modification to PIM-SM, a multicast router is able to receive registration from nearby group members and redirect passing-by join requests to them. Due to the multicast router's support, TOMOON organizes its group members into an overlay multicast tree efficiently, which matches the physical network topology well. Since we don't depend on routers to multicast data packets, the overlay tree can also cover the unicast networks and thus has a good support for the heterogeneous network environment.
\end{abstract}

\section{Introduction}

Most of the current overlay multicast solutions (e.g. Narada 3], Nice 2]) share some common drawbacks. First, they can't adapt the overlay to the physical network very well. Thus it is hard to guarantee the cost-efficiency of data transmission in all cases. Second, they force end-hosts to assume too much responsibility in overlay construction, which makes the protocol too complicated to serve as a general platform for other applications. Last, since the recovery from overlay partition can be very slow, it is hard to maintain a stable performance in case of node failures. All these issues result from a common design principle. That is to remain the network layer as simple as possible and refuse to consider any support from the network no matter whether it is available or not.

Our contribution in this paper is to address all these issues by proposing a topology-aware overlay multicast approach, which is supported by IP multicast networks. The key point of our approach is to adopt the overlay network for actual data transmission while exploiting IP multicast routers to optimize the overlay construction. This method has several advantages over traditional overlay multicast schemes. With support of the multicast-enabled network layer, the 
task of overlay topology optimization can be drastically simplified. The complexity of the dynamic membership management is also reduced to a large extent. Meanwhile, end-host based multicast eliminates most of the problems related to IP multicast. Since the multicast routers may be deployed incrementally in our approach, the overlay can easily scale up to support large-sized group communications among heterogenous networks, where not all routers are multicastcapable. Besides the scalability issue and the address collision, overlay multicast also provides a viable solution to the commercial issue, which is difficult in IP multicast. Therefore the two multicast techniques, which seem to contradict at a first glance, are merged to produce an efficient multicast framework in this paper. Hereinafter, we name it by TOMOON, which stands for Topology-aware Overlay Multicast Over heterOgeneous Networks.

The rest of the paper is organized as follows: In Sect 2 we define the network model for TOMOON and analyze the mechanism of IP multicast. The detailed protocol is explained in Sect 3 , which describes our modification to the PIM-SM[5] protocol and the tree construction algorithm. The performance of TOMOON is evaluated through simulations in Sect 4 . Finally we draw conclusions in Sect 5 .

\section{Network Models}

\subsection{Physical Network Model}

Without loss of generality, we model the underlying network in question by a directed graph $\mathrm{G}(\mathrm{V}, \mathrm{E})$ with the vertices representing routers or hosts and the edges standing for links. A host is attached to only one router while a router's node degree is more than one. Each edge is assigned a weight to indicate the link's cost or latency. We assume that each node here belongs to an autonomous system (AS) that is either a multicast domain or a unicast domain. All routers of a multicast domain support intra-domain IP multicast. To make the following description easier, we now define some concepts, which will be used throughout the paper.

Definition 1. A path from node $A$ to node $B$, denoted by $P(A, B)$, is a sequence of routers comprising a shortest path from $A$ to $B$ according to the underlying unicast routing protocol. $P(A, S)$ is also called a request path of node $A$ if node $S$ is a data source. Since a unique data source is usually presumed, the request path of $A$ is also denoted by RPath $(A)$.

Definition 2. Node $B$ is close to the request path of node $A$ if at least one node of RPath $(A)$ is within a certain distance from node $B$ and they are located in the same multicast domain. This distance is also called a capture range, which is determined by the physical network settings around node $B$.

An outline of the physical network model is shown in Fig. 1 In this figure, all router nodes are marked with capital letters while the gray nodes represent end hosts. Suppose that node $s$ is the unique source and all other hosts are receivers 
of the same group. We use the dotted lines to indicate the request paths of $R P a t h(a)$ and RPath(b), which originate from $a$ and $b$ respectively and point to node $s$. If the capture range is just one hop, only $c$ and $g$ are close to $R P a t h(b)$. Since $e 1$ and $e 2$ are located in a unicast domain, they are not close to RPath(b) even if they are just one hop away from the router $E$.

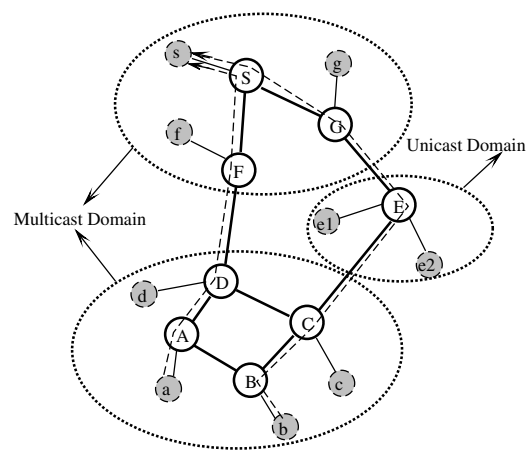

Fig. 1. Physical network model

\subsection{Introduction to PIM-SM}

Multicast forwarding entry. From a multicast router's perspective, the task of any routing protocol is to build a routing table with forwarding entries for all groups. A forwarding entry for the PIM-SM multicasting is denoted by a quaternary tuple of (S,G,iif,oif), which applies to all packets originated from source S and destined for group G. The other two fields, namely iif and oif, refer to input interface list and output interface list respectively. The construction of such a forwarding entry and its usage is explained as follows.

Control Path. For simplicity, we only consider the source specific multicast in PIM-SM. Here PIM-SM routers have to deal with two protocols in order to create the forwarding entries for a $(\mathrm{S}, \mathrm{G})$ pair. First, the designated router of the receiver will receive an IGMP[1] source specific membership report from the host, which carries the $(\mathrm{S}, \mathrm{G})$ information. Then it turns this membership report into a PIM-SM join request and unicasts it towards the source S. All routers receiving this request should build an entry like ( $\mathrm{S}, \mathrm{G}$, iif, oif ). The input interface for receiving the IGMP membership report or the PIM-SM join request is added to oif while the output interface for sending the join request is added to iif. In this way, the multicast forwarding entry is installed on all PIM-SM routers involved in the group session, which form a shortest path tree from the sender to all receivers.

Data Path. Once the shortest path tree is established, the forwarding entry comes into play for packet multicasting. Whenever a router on the distribution 
tree receives a multicast packet sent from source $\mathrm{S}$ to group $\mathrm{G}$, it will first check whether the input interface of this packet is exactly the one in iif. If the packet does come in through the correct interface, a copy of it is sent out through each interface listed in oif. The forwarding process is done in a top-down manner from the source's designated router to all leaf routers on the tree.

\section{Details of TOMOON}

\subsection{Multicasting Join Requests}

Multicast-capable routers play an important role in our overlay tree construction. Briefly speaking, TOMOON depends on those routers on the new joiner's request path to multicast the join request towards their respective neighbor members so that the new member can find a suitable parent from the tree quickly. While a PIM-SM router is totally qualified to assume such a task from a functional perspective, no existing protocol is designed for this purpose. Therefore we need to introduce some modifications to PIM-SM so that the routers will behave as we expect them to.

Multicast Forwarding Entry. One basic function of the multicast forwarding entry is to determine whether a packet belongs to a specific group so that it can be multicasted accordingly. Unlike the situation in source specific IP multicast, where all data packets of the same group have a common source-destination pair like $(S, G)$, the join requests for the same group session originate from different joiners and thus have different source addresses. Since TOMOON is designed for single source multicast, we will aggregate the join requests of the same session by their destination address/port pair like $(\mathrm{S}, \mathrm{P}) . \mathrm{S}$ is in fact the address of the overlay tree's root. The inclusion of the port number $\mathrm{P}$ allows for multiple overlay trees rooted at the same node. Therefore the multicast forwarding entry for multicasting the join requests is a ternary tuple like $(\mathrm{S}, \mathrm{P}$, oif $)$. The field oif has a similar meaning as we described previously. Now we are going to explain the installation and application of these entries on routers.

Membership Registration. In order to capture the wanted messages on nearby routers, an in-tree member $M$ sends a membership report toward the tree root with a limited value of TTL. Due to the small TTL, only those routers close to $\mathrm{M}$ will receive it. Once a router $\mathrm{R}$ has received such a report, which indicates a (S, P) pair, it will initiate a forwarding entry building process, which is described in detail by the pseudo-code routine in Fig. 2 ,

Forwarding Join Request. The process of multicasting join requests is straightforward once the forwarding entry is calculated as mentioned above. When a join request destined for the session $(\mathrm{S}, \mathrm{P})$ arrives at a router where a corresponding forwarding entry is installed, a copy of it is sent through each of the interfaces in oif except the input interface of the request. If the oif field only has two interfaces and the request is received from one of them, then the 


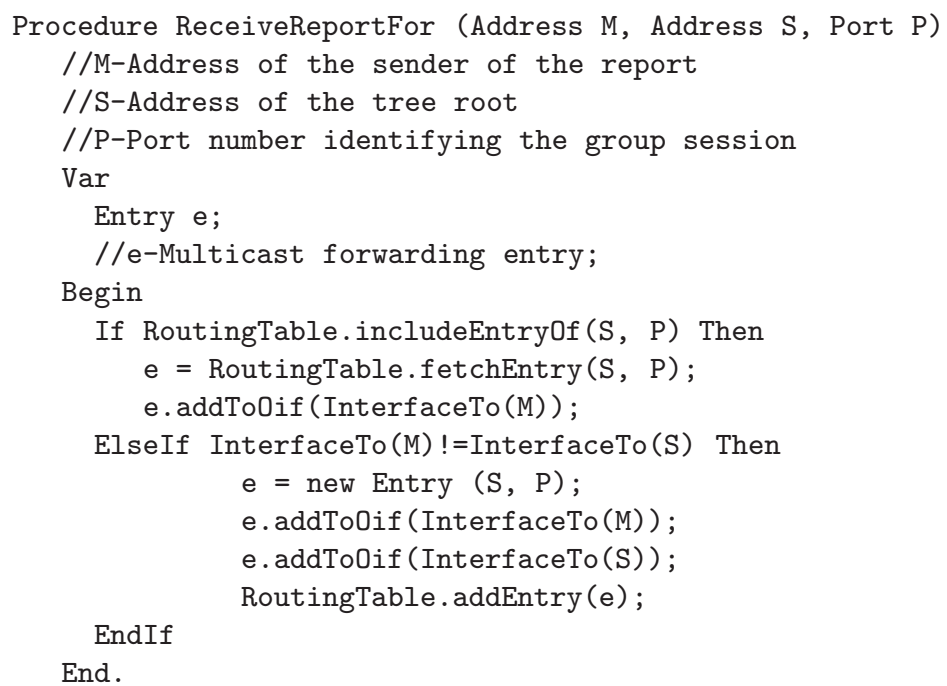

Fig. 2. Algorithm for building forwarding entries

transmission will reduce to a unicast forwarding. This stipulation ensures that all members registered at the routers will receive exactly one copy of the request during the multicasting.

\subsection{Tree Construction and Maintaining}

Member Joins. Suppose that each new member J knows the address/port pair $(\mathrm{S}, \mathrm{P})$ of the data source before its participation. Then a request packet is generated with $(\mathrm{S}, \mathrm{P})$ as its destination address/port pair. As we mentioned above, if the join request traverses the capture ranges of other in-tree members, the data source $\mathrm{S}$ along with those members close to the request path will be informed of this new joiner. Each of them will respond to J as long as they have sufficient residual fan-out degrees to accept a new child in the overlay tree. Each response indicates a candidate parent. The next step is to choose a best one from them as J's formal parent in the tree.

The specific criteria for choosing a best parent node could vary from one application to another. For simplicity, we order that the candidate closest to the joiner become its parent. If multiple candidates are qualified, the one closest to the data source is selected. This can be done by using tools like ping or traceroute. Meanwhile, a list of the candidate parents is kept for later use.

Topology Adjustment. As far as tree-based multicasting is concerned, a key reason for topology adjustment is that some in-tree members would like to be connected to newcomers rather than their current parents. This idea is explained in Fig. 3. In this figure, node $d$ is close to the request path of node $a$. However, node $a$ joins the tree before node $d$, which leads to a tree structure like 


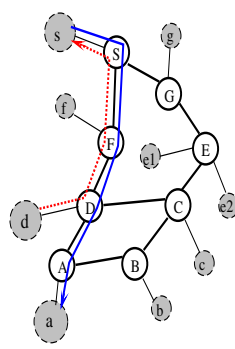

(a)

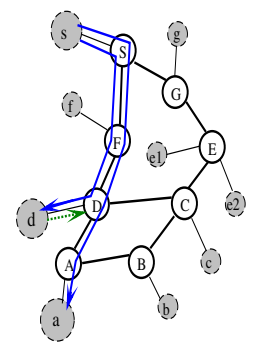

(b)

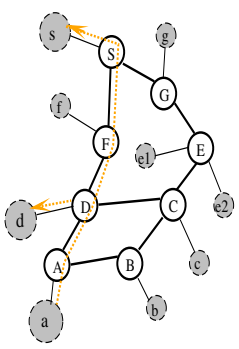

(c)

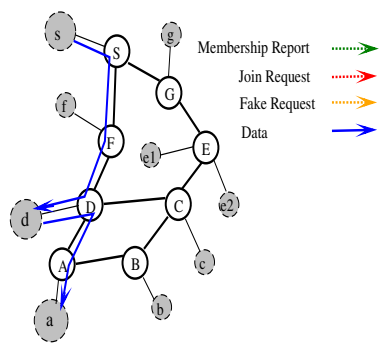

(d)

Fig. 3. Topology adjustment

Fig. 3(b). While the tree in Fig. 3(d) is more cost-efficient in terms of bandwidth consumption, the join process of $d$ doesn't provides a mechanism to inform the downstream members of the upstream newcomers.

Our solution is to have downstream in-tree members send a fake join request towards the data source periodically. The fake request has the same destination address/port pair as a true one has. Since the newcomer $d$ is close to the request path of node $a$, it can capture this fake request as shown in Fig. 3(c). Except the newcomer, other members will not respond to a fake request of a familiar sender. Then the tree could be transformed like Fig. 3(d) for a better shape.

Partition Recovery. If a failure has occurred, the abandoned children follow a join-and-adjust strategy. In other words, the child first tries to make a connection with the best candidate parent in its current list to join the group. Once the overlay link is established, an adjustment process goes on to optimize the overlay topology after the node failure. Due to the candidate parent list, an abandoned child node can find the new parent immediately while still maintaining a good performance of the overlay transmission. Therefore the impact of node failures is reduced to the minimum.

\section{Evaluation}

In this section, we evaluate the TOMOON protocol using simulations. The main purpose of the simulations here is to investigate how much a TOMOON tree matches the topology of the underlying network. For this purpose, we measure the tree cost in our simulations for a quantified result.

Two benchmark protocols we adopt for comparison with TOMOON are PIMSM and TAG 4]. As we introduced in Sect 2.2, we consider the former as a lowerbound benchmark. We also choose TAG for comparison because it is similar to TOMOON in its design intention. We adopt the Waxman approach [6] to generate a topology to mimic the Internet for our experiments. The fan-out degree of each node in the graph has an upper bound of 6 . The cost and delay of each link is set to the same value, which varies from 1 to 5 for intra-domain links and from 6 to 
10 for inter-domain links. To evaluate the performance of our protocol in different network environments, we repeat our simulations in two different scenarios:

1. Physical links have symmetric cost.

2. Physical links have asymmetric cost.

\subsection{Simulation Results and Discussions}

In the first experiment, we assume that all domains support IP multicast. Whenever a node joins the group in TOMOON, it will broadcast its membership report throughout its domain to capture other join requests passing by. We change the group size from 20 to 100 . Fig. 4 shows the tree cost for two scenarios.
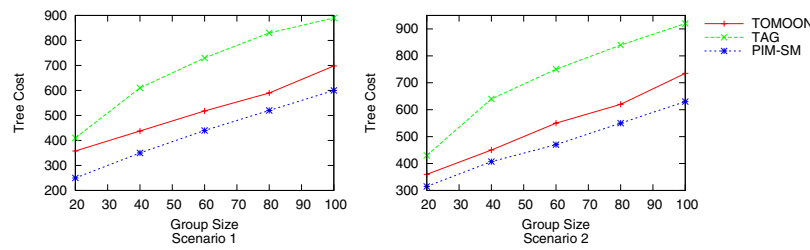

Fig. 4. Group size VS tree cost

From this figure, we can infer that the performance of TOMOON is better than TAG and approaches that of PIM-SM as the membership increases. We give the reasons for this result from two aspects. First, as an increasing number of nodes join the group in TOMOON, it is much easier for a new member to find a parent in its close neighborhood. In other words, the extra cost for accepting a new member into the group decreases when the membership increases. Second, the overlay of TAG is not adapted to the underlying network topology in all situations. Although the parent and its new child share a longest segment on the routes from the source to each of them in TAG, it is still possible that the overlay link between these two nodes is longer than other alternatives. Thus TAG is less aggressive than TOMOON in saving the tree cost.

In the second experiment, we assume that only a part of domains support IP multicast. It is much closer to the situation of the real Internet. We select two
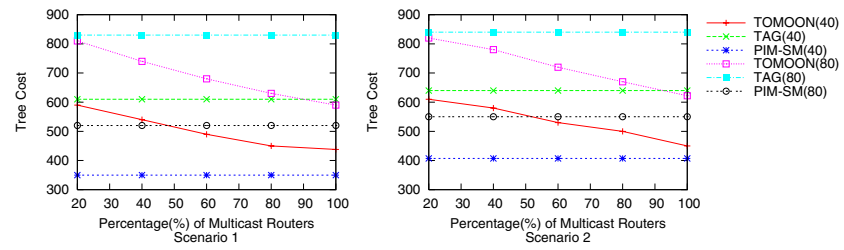

Fig. 5. Percentage of multicast domains VS tree cost 
fixed group sizes(40 and 80) and adjust the percentage of multicast domains from $20 \%$ to $100 \%$ to study TOMOON's performance in different environments. The growing percentage of multicast domains is transparent to other two protocols

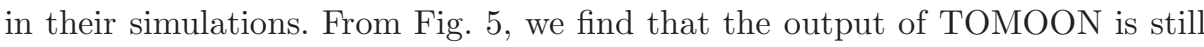
acceptable even when the multicast domains account for a small percentage in the underlying network.

\section{Conclusion}

In this paper, we propose TOMOON as a novel overlay multicast approach, which exploits the support from IP multicast routers to construct a topologyaware overlay tree. Compared with IP multicast and other overlay multicast schemes, our approach has the following notable advantages :

1. A TOMOON tree is compatible with the physical network. With the help of multicast routers, inefficient branches in a multicast tree are eliminated.

2. TOMOON makes the membership management much easier in overlay multicast. In other words, TOMOON can deal with member joins and leaves efficiently.

3. TOMOON encourages ISPs to provide better network layer support. Since the multicast routers can capture consumers' requests of various network services, ISPs are willing to build multicast domains for expectable commercial benefits.

\section{References}

1. Cain, et. al.: Internet Group Management Protocol, Version 3, RFC 3376, October 2002

2. S. Banerjee, B. Bhattacharjee, and C. Kommareddy: Scalable application layer multicast. Proc. ACM Sigcomm, Aug. 2002

3. Y.-H. Chu, S. G. Rao, and H. Zhang: A Case for End System Multicast. IEEE J. Select. Areas Commun, VOL. 20, NO. 8, OCTOBER 2002.

4. Kwon, Minseok, and Sonia Fahmy: Path-aware overlay multicast Computer Networks 2005(47), pp. 23-45.

5. Estrin, et. al.: Protocol Independent Multicast-Sparse Mode (PIM-SM): Protocol Specification", RFC 2362, June 1998

6. B.M. Waxman: Routing of multipoint connections.IEEE J. Select. Areas Commun., vol.6, no. 9, Dec. 1988. 